\title{
Pattern Generation and the Control of Nonlinear Systems
}

\author{
Roger W. Brockett, Fellow, IEEE
}

\begin{abstract}
Many important engineering systems accomplish their purpose using cyclic processes whose characteristics are under feedback control. Examples involving thermodynamic cycles and electromechanical energy conversion processes are particularly noteworthy. Likewise, cyclic processes are prevalent in nature and the idea of a pattern generator is widely used to rationalize mechanisms used for orchestrating movements such as those involved in locomotion and respiration. In this paper, we develop a linkage between the use of cyclic processes and the control of nonholonomic systems, emphasizing the problem of achieving stable regulation. The discussion brings to the fore characteristic phenomena that distinguish the regulation problem for such strongly nonlinear systems from the more commonly studied linear feedback regulators. Finally, we compare this approach to controlling nonholonomic systems to another approach based on the idea of an open-loop approximate inverse as discussed in the literature.
\end{abstract}

Index Terms-Inverse systems, Lie brackets, nonlinear control, pattern generation, regulation, stabilization.

\section{INTRODUCTION}

$\mathbf{I}$ $\mathrm{N}$ THIS PAPER, the word "pattern" is used to denote a collection of periodic, or nearly periodic, vector valued functions of time. The choice of words is inspired by language used in neuroscience and biological motor control where approximate periodicity is common and true periodicity rare. In fact, for the applications we have in mind it is only the qualitative properties of the functions that matter; successive cycles need not have exactly the same waveform and/or the same period as long as they possess the appropriate general shape. We will argue that the need for, and use of, patterns is closely related to nonlinear controllability and that the operation of important classes of such systems is entirely dependent on nonlinear effects. In the literature this effect is associated with phenomena such as Berry's phase [1], rectification and area rules, [2], etc. We adopt a point of view that unifies and explains these problems in terms of concepts from geometric nonlinear control.

The main points to be developed are as follows.

1) In those applications where the successful operation of a system depends on cyclic motion, there is often an un-

Manuscript received September 1, 2002; revised December 4, 2003 and May 8, 2003. Recommended by Guest Editors W. Lin, J. Baillieul, and A. Bloch. This work was supported in part by the Army Research Office under Grant DAAG 55-97-1-0114, by DARPA under Grant DAAH 04-96-1-0445, by the National Science Foundation under Yale prime CCR 9980058, by the Army Research Office under Boston University prime GC169369 NGD, by the National Science Foundation under Grant EIA 0218411, and by DARPA under Stanford Prime PY-1606.

The author is with the Division of Applied Science, Harvard University, Cambridge, MA 02138 USA (e-mail: brockett@hrl.harvard.edu).

Digital Object Identifier 10.1109/TAC.2003.817927 derlying strongly nonlinear subsystem whose controllability depends on the nonintegrability of a family of vector fields.

2) To obtain suitable tracking and regulation properties with such systems, it is usually necessary to generate and shape patterns, giving them the correct amplitudes, frequencies, and relative phases. Although the basic shapes can be generated in an open-loop way, feedback is essential to accommodate changing loads.

3) When attempting to solve feedback regulation problems in which nonintegrability is important, it is often insightful to identify an appropriate output, relative to which the system is lossless. Here, this means focusing attention on input-output systems taking the form of a nonlinear integrator

$$
\dot{x}=G(x) u \quad y=G^{T}(x) x .
$$

Such systems are lossless relative to the internal energy function $x^{T} x / 2$.

Common control problems ranging from the control of internal combustion engines to the regulation of respiration in mammals are profoundly nonlinear. In many cases of interest, one can linearize about a steady state, usually a periodically varying solution, and get some insight into the dynamics. However, this sort of analysis provides no explanation as to why the system was designed to use periodic motion in the first place. For example, it has long been recognized that provision for pattern generation is an important part of the neural circuitry used to generate and control various animal movements such as walking, breathing, blood circulation, peristalsis, etc., yet linear theory is silent about the need for this mode of control. Parametric amplifiers and switched capacitor filters provide examples from electrical engineering in which pattern generation is essential. Applications of particular technological significance include rotating electrical machinery [3] and the use of orchestrated periodic switching to transform direct current at one voltage to direct current at a different voltage [4]. In the domain of mechanics, vibratory motors [2] provide a class of highly nonlinear examples. Recently, interest has been focused on this area because of robotic applications involving wheeled vehicles and object manipulation, and because of spacecraft control problems involving nonholonomic effects. Specific problems discussed in the literature include the control of unicycles, parallel parking, control of autonomous vehicles, and the steering of tractor-trailer systems.

A number of different points of view on pattern generation are to be found in the literature. Prominent among these are the physical point of view placing emphasis on the role of energy, 
momentum, etc. and the cybernetic point of view placing emphasis on the flow of signals necessary for stabilization, motion planning, etc. The first of these is exemplified by the equations of an electrical machine, relating the amplitude of the field current and the angular velocity of the shaft and the equations of motion for a satellite being controlled by momentum wheels. The second point of view is more prominent in neuroscience where pattern generators are often studied without considering the dynamics of the elements being controlled. However, even in this literature important experiments, such as those of [5], show a strong link between physics and pattern generation. The development here is closely tied to the physical point of view.

\section{EXAMPLES}

There is a large literature on driftless, multi-input systems of the form $\dot{x}=G(x) u$. Our starting point is the related input-output system

$$
\dot{x}=G(x) u \quad y=G^{T}(x) x .
$$

A basic property of such systems is that they are lossless in the sense that

$$
\frac{1}{2} \frac{d}{d t} x^{T} x=x^{T} G(x) u=\langle y, u\rangle
$$

and, thus, feedback controllers realized by connecting $y$ to $u$ via a passive system will result in a closed-loop system having bounded trajectories. For the problems considered here, the structure of $G$ suggests decomposing the input space into two parts so that we may write

$$
\dot{x}=G_{1}(x) u_{1}+G_{2}(x) u_{2} \quad y_{1}=G_{1}^{T}(x) x \quad y_{2}=G_{2}^{T}(x) x .
$$

If we apply partial feedback in the form $u_{2}=-\phi\left(y_{2}\right)$, we get a system with a drift term

$$
\dot{x}=-G_{2}(x) \phi\left(G_{2}^{T}(x) x\right)+G_{1}(x) u_{1} \quad y_{1}=G_{1}^{T}(x) x
$$

which is still passive in the $u_{1}$ to $y_{1}$ channel. In the situations to be discussed here, the systems are not only controllable using ( $u_{1}, u_{2}$ ) but are even controllable using $u_{1}$ alone. The problem to be addressed is that of finding a control law for $u_{1}$ which will maintain the value of $y_{2}$ at some desired value with $y_{1}$ being periodic with some specified average value.

\section{A. Basic Model}

The most elementary, and in a limited sense canonical, model for the phenomena to be studied here has the input-to-state description

$$
\dot{x}_{1}=u_{1} \quad \dot{x}_{2}=u_{2} \quad \dot{x}_{3}=x_{1} u_{2}-x_{2} u_{1}+u_{3} .
$$

We associate outputs with this system according to the rule $y=$ $G^{T}(x) x$, i.e.,

$$
y_{1}=x_{1}-x_{3} x_{2} \quad y_{2}=x_{2}+x_{3} x_{1} \quad y_{3}=x_{3} .
$$

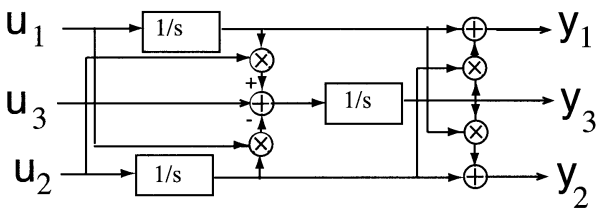

Fig. 1. Block diagram for the basic input-output system for $m=2$.

(See Fig. 1). As remarked before, this system is lossless in the sense that $(1 / 2)(d / d t) x^{T} x=\langle y, u\rangle$. If we consider $x_{3}$ as defining a skew-symmetric matrix via

$$
X(t)=\left[\begin{array}{cc}
0 & x_{3} \\
-x_{3} & 0
\end{array}\right]
$$

then these equations can be written more succinctly, and in a form that generalizes to any number of dimensions

$$
\begin{aligned}
& \dot{x}=u \quad \dot{X}=x u^{T}-u x^{T}+U \quad \text { (the control system) } \\
& y=x-X x \quad Y=X \quad \text { (the output map). }
\end{aligned}
$$

This input-output system, having input pair $(u, U)$ and output pair $(y, Y)$, is passive relative to the internal energy function $(1 / 2) x^{T} x+(1 / 4) \operatorname{tr}\left(\mathrm{X}^{\mathrm{T}} \mathrm{X}\right)$ in the sense that

$$
\frac{d}{d t}\left(\frac{1}{2} x^{T} x+\frac{1}{4} \operatorname{tr}\left(\mathrm{X}^{\mathrm{T}} \mathrm{X}\right)\right)=\langle y, u\rangle+\frac{1}{2}\langle X, U\rangle .
$$

The control equations involving just $(x, X, u)$ were apparently first singled out for study in [6] and [7]. Prior work justifies the point of view that this system is to first bracket controllable systems what linear systems are to linearly controllable systems; see [7, Th. 1]. It is the prototype for first bracket controllable systems. The input-output system appearing here does not seem to have been studied before.

Rather than appearing in this elemental form, it often happens that the basic model is embedded in a larger system that incorporates additional feedback paths and/or complicating dynamical effects. A simple example of how this system might be altered by feedback is to let $U$ equal $-X$ so as to get

$$
\dot{x}=u \quad \dot{X}=-X+x u^{T}-u x^{T} .
$$

\section{B. Rotating Electrical Machinery}

The conversion of energy associated with the flow of current in a wire, into energy associated with the rotation of a shaft has been identified with nonholonomic systems since [3]. The Lorentz force $f=i \times B$ from electromagnetic theory occupies a central role. If one works in a coordinate frame that rotates with the rotor of an electrical machine then in terms of the components of the magnetic field, $x$ and $y$ and the current through the armature coils, $u, v$, the system satisfies

$\dot{x}_{1}=-\alpha x_{1}+u_{1} \quad \dot{x}_{2}=-\alpha x_{2}+u_{2} \quad \dot{\omega}=-k \omega+x_{1} u_{2}-x_{2} u_{1}$

where $\alpha$ characterizes the electrical resistance in the coils, $\omega$ is the angular rotation rate and $k$ represents a coefficient of viscous friction. Obviously units have been normalized. The motor 
equations are obtained from the basic system by using feedback to replace $u_{i}$ by $u_{i}-k x_{i}$, and $U$ by $-X$.

\section{Internal Combustion Engines}

The ability of an internal combustion engine to maintain an angular velocity in the presence of an opposing torque depends on a cyclic process of the type we are discussing. Let $\dot{\theta}$ denote the angular velocity of the crankshaft. Let $x_{1}$ denote the pressure of the gas acting on the piston and let $x_{2}$ denote the volume of this gas in the cylinder. In this case, the variable $x_{2}$ is a periodic function of the crank shaft angle $\theta$. Say $x_{2}=\phi(\theta)$. Thus, the equations must be augmented with an equation for $\theta$. We assume that the pressure is directly controllable (through the supply of fuel and air) and call this control $u_{1}$. We further assume that the load can be modeled by viscous friction. The equations can be written as

$$
\begin{aligned}
\dot{x}_{1} & =u_{1} \quad \dot{x}_{2}=u_{2}=\frac{d \phi}{d \theta} \dot{\theta} \\
\ddot{\theta} & =-\dot{\theta}+u_{1} x_{2} .
\end{aligned}
$$

Although this is less symmetrical than the rotating electrical machine example, the qualitative properties are essentially the same.

\section{Biological Phenomena}

In 1911, Brown [8] published a paper in the Proceedings of the Royal Society with the wonderful title, "Intrinsic factors in the Act of Progression of the Mammal." The substance of his paper has to do with patterns associated with locomotion and this topic has continued to be studied vigorously to this day. Of particular interest in this setting is the fact that both the frequency and the amplitude of the oscillation is used in regulation. For example, it is a familiar fact that as one walks faster both the length of the stride and the frequency of the steps increase. Likewise, when faced with the need for a higher respiratory rate, humans both increase the volume of air processed per breath and the number of breaths per minute. Data on the locomotion problem is cited in [9] and breathing is discussed, for example, in [10].

\section{GENERALITIES ON FIRST BRACKET CONTROLLABLE SYSTEMS}

Let $u$ be a $m$-dimensional and let $x$ be $n$-dimensional and consider

$$
\dot{x}=G(x) u=\sum_{i=1}^{m} g_{i}(x) u_{i} .
$$

As is well known, when the vectors $g_{i}$ are differentiable a suitable number of times, the controllability of such systems is best studied by thinking of the $g_{i}$ as defining vector fields and focusing attention on the way in which the Lie brackets

$$
\left[g_{1}, g_{2}\right]=-\left[g_{2}, g_{1}\right]=\frac{\partial g_{2}}{\partial x} g_{1}-\frac{\partial g_{1}}{\partial x} g_{2}
$$

enlarge the vector space spanned by vectors $g_{1}, g_{2}, \ldots, g_{m}$. We limit our discussion to the situation in which the $m$ vectors $\left\{g_{i}\right\}$ together with the $m(m-1) / 2$ vectors generated by taking their first brackets $\left.\left\{\left[g_{1}, g_{2}\right],\left[g_{1}, g_{3}\right], \ldots,\left[g_{m-1}, g_{m}\right]\right\}\right\}$ span $\mathbb{R}^{n}$. Such systems will be called first-bracket controllable. At a generic point $x_{0}, G\left(x_{0}\right)$ will have a range space that is at most $m$-dimensional. The number of additional linearly independent directions coming from the bracket terms can not exceed the number of linearly independent brackets which is $m(m-1) / 2$. Thus, we see that if the system is first-bracket controllable $n \leq m(m+1) / 2$.

If the system is controllable in a neighborhood of $x_{0}$ then $G\left(x_{0}\right)$ must be nonzero. Using linear transformations $x \mapsto P x$ and $u \mapsto M u$, we can arrange matters so that

$$
G\left(x_{0}\right)=\left[\begin{array}{ll}
I & 0 \\
0 & 0
\end{array}\right] .
$$

If $x$ takes on values in $\mathbb{R}^{n}$ and $u$ in $\mathbb{R}^{m}$ and if $n \geq m$ then generically the identity matrix will be $m$ by $m$ so that

$$
G\left(x_{0}\right)=\left[\begin{array}{l}
I \\
0
\end{array}\right] .
$$

Assuming that the generic condition is satisfied and that the appropriate changes of coordinates have been used, we can expand the $g_{i}$ in a Taylor series about $x_{0}$. For the first $m$ coordinates

$$
g_{i}(x)=e_{i}+A_{i}\left(x-x_{0}\right)+\phi_{i}(x), \quad i=1,2, \ldots, m
$$

where $e_{i}$ is the $i$ th standard basis element and $\phi_{i}$ is $O\left(\left(x-x_{0}\right)^{2}\right)$. For the remaining coordinates

$$
\dot{x}_{l}=G_{l}(x) u \quad G_{l}\left(x_{0}\right)=0 .
$$

With some difficulty one can show (see the proof of [7, Th. 1]) that through a redefinition of the variables one can arrange matters so that the first $m$ rows of the $A_{i}$ are all zero and the remaining rows are such that we have

$$
\begin{aligned}
\dot{x}_{i} & =u_{i}+\sum \phi_{i j}(x) u_{j} \quad \phi_{i j}=O\left(x^{2}\right), \quad 1 \leq i \leq m \\
\dot{x}_{i} & =\sum x_{j} s_{i j k} u_{k}+\sum \psi_{i j}(x) u_{j} \\
\psi_{i j} & =O\left(x^{2}\right) \quad m+1 \leq i \leq n
\end{aligned}
$$

with the trilinear form $s_{i j k}$ being skew-symmetric in the last two indexes. From this, we see that if we neglect the higher order terms then locally the system can be identified with a subsystem of

$$
\dot{x}=u \quad \dot{X}=x u^{T}-u x^{T}
$$

obtained from it by (possibly) ignoring some of the equations defining the entries of $Z$.

The use of temporal patterns to achieve a desired form of behavior for such systems can often be traced to the following situation. Consider a system

$$
\dot{x}(t)=g_{1}(x(t)) u_{1}(t)+g_{2}(x(t)) u_{2}(t) \quad x(0)=0
$$

with $g_{1}(0)=e_{1}$ and $g_{2}(0)=e_{2}$. It is clear that we can use the controls to adjust $x_{1}$ and $x_{2}$ at will. However, it is usually possible to do more by means of an indirect procedure. If we 
use $u_{1}$ and $u_{2}$ to take $x_{1}$ and $x_{2}$ around in a closed loop then typically there will be some change in the remaining variables. In fact, the procedure for computing this change is by now well known. If we let $u_{1}(t)=a \cos 2 \pi t$ and $u_{2}(t)=a \sin (2 \pi t)$ then $x$ does not remain at the origin but is displaced

$$
x(1)-x(0)=\left.\frac{\pi a^{2}}{4}\left[g_{1}, g_{2}\right]\right|_{x_{0}}+O\left(a^{3}\right) .
$$

One can get a good intuition for the proof by replacing the trigonometric functions by the simpler functions, $u(t)=a$. $\operatorname{sgn}(\sin 2 \pi t)$ and $v(t)=a \cdot \operatorname{sgn}(\cos 2 \pi t)$ and doing a careful (tedious) calculation, using repeatedly the fact that to second order in $t$ the solution $\dot{x}=f(x)$ is

$$
x(t)=x(0)+f(x(0)) t+\left.\frac{1}{2} \frac{\partial f}{\partial x}\right|_{x(0)} f(x(0)) t^{2}+O\left(t^{3}\right) .
$$

It is worth repeating that even though the average value of $u_{1}$ and $u_{2}$ is zero, the pattern of variation about 0 gives rise to a definite displacement as revealed by a second order calculation.

This result can be recast to avoid the specific role played by trigonometric functions. Notice that if the integral over $[0,1]$ of both $u$ and $v$ is zero, then the locus of points traced out for $0 \leq t \leq 1$

$$
\left(\left(x_{1}(t), x_{2}(t)\right)=\left(\int_{0}^{t} u_{1}(\sigma) d \sigma, \int_{0}^{t} u_{2}(\sigma) d \sigma\right)\right.
$$

generates a closed curve in $\mathbb{R}^{2}$ and this closed curve defines some area. We consider this area to have a sign, positive if the curve in $\left(x_{1}, x_{2}\right)$-space is traversed in the counterclockwise direction. With this understanding we can assert that $\Delta=$ $x(1)-x(0)$ is approximately $\left[g_{1}, g_{2}\right]$ evaluated at $x=x(0)$ times the area defined by the closed curve given by the graph of $\left(x_{1}, x_{2}\right)$. When $x_{1}$ and $x_{2}$ are periodic, the curve in $\mathbb{R}^{2}$ is traced out repeatedly. If it is circular, the average of the time derivative of $x$ is the rate at which $\left(x_{1}, x_{2}\right)$ sweeps out area multiplied by the bracket evaluated at 0 . Many examples of "area rules" appear in engineering, physics and mathematics; see [1] and [2]. We observe that the basic properties of the nonholonomic integrator are qualitatively unchanged if we replace the equation for $x_{3}$ with $\dot{x}_{3}=\phi\left(x_{1}, x_{2}\right) u_{1}-\psi\left(x_{1}, x_{2}\right) u_{2}$ as long as the spanning condition $\left(\partial \phi / \partial x_{2}\right) \neq\left(\partial \psi / \partial x_{1}\right)$ holds.

The main point of this paper is to show how the oscillations needed to obtain the area can be generated spontaneously and in a stable way by feedback control laws of the general form

$$
u=\left(\Omega+Q\left(x-x_{d}\right)\right) G_{1}^{T} x
$$

where $\Omega=-\Omega^{T}$ and $Q\left(x-x_{d}\right)=Q^{T}\left(x-x_{d}\right)$.

\section{Oscillations FOR Set Point CONTROL}

We now begin to address the main questions of interest, building toward a series of results on set point regulation using temporal patterns. The results to be derived assert the existence of feedback control laws providing stable regulation of a limit cycle for first bracket controllable systems. This section considers the problem of establishing and regulating patterns in $\left(x_{1}, x_{2}\right)$-space suitable for maintaining a steady nonzero value for $x_{3}$ in the face of a "load." More precisely, we want to maintain $x_{3}$ at some specified value $e>0$ for the system

$$
\dot{x}_{1}=u_{1} \quad \dot{x}_{2}=u_{2} \quad \dot{x}_{3}=-x_{3}+x_{1} u_{2}-x_{2} u_{1} \text {. }
$$

This is typical in a situation where $x_{3}$ is, for example, a flow rate which is to be maintained at some specific positive value in opposition to a resistance represented by the $-x_{3}$ term. The problem is that of finding $u_{1}\left(x_{1}, x_{2}, x_{3}\right)$ and $u_{2}\left(x_{1}, x_{2}, x_{3}\right)$ such that the steady state solution of this system of equations is a stable oscillation in the $\left(x_{1}, x_{2}\right)$ variables maintaining $x_{3}$ at the constant value $e$. Observe that if $\beta, \omega$ and $e$ are positive real numbers, then the choices

$$
\begin{aligned}
& u_{1}=-\omega x_{2}-\beta\left(x_{3}-e\right) x_{1} \\
& u_{2}=\omega x_{1}-\beta\left(x_{3}-e\right) x_{2}
\end{aligned}
$$

will cause the $\left(x_{1}, x_{2}\right)$ variables to traverse a circle and their contribution to the right-hand side of the third equation will be such as to offset the effect of the $-x_{3}$ term. The terms $\beta(e-$ $\left.x_{3}\right) x_{1}$ and $\beta\left(e-x_{3}\right) x_{2}$ serve to adjust the amplitude of the oscillation unless the amplitude of $\left(x_{1}, x_{2}\right)$ is exactly what is needed to make $x_{3}=e$. The following theorem addresses the question of stability.

Theorem 1: If $x$ and $u$ are related by

$$
\frac{d}{d t}\left[\begin{array}{l}
x_{1} \\
x_{2} \\
x_{3}
\end{array}\right]=\left[\begin{array}{c}
u_{1} \\
u_{2} \\
x_{1} u_{2}-x_{2} u_{1}-\alpha x_{3}
\end{array}\right]
$$

with $\alpha>0$, then there exists $\omega \in \mathbb{R}$ such that for every positive $\beta$ and every $e>0$, the control law

$$
\left[\begin{array}{l}
u_{1} \\
u_{2}
\end{array}\right]=\left[\begin{array}{c}
-\omega x_{2}+\beta\left(e-x_{3}\right) x_{1} \\
\omega x_{1}+\beta\left(e-x_{3}\right) x_{2}
\end{array}\right]
$$

defines a closed-loop system that admits a one-parameter family of periodic solutions

$$
x_{p}(t)=\left[\begin{array}{c}
\sqrt{\frac{\alpha e}{\omega}} \cos (\omega t+\phi) \\
\sqrt{\frac{\alpha e}{\omega}} \sin (\omega t+\phi) \\
e
\end{array}\right], \quad 0 \leq \phi<2 \pi .
$$

Each such periodic solution is stable and all solutions starting off the line $x_{1}=x_{2}=0$ approach the circle defined by $M_{\infty}=$ $\left\{\left(x_{1}, x_{2}, x_{3}\right) \mid\left(x_{1}^{2}+x_{2}^{2}=\alpha e / \omega ; x_{3}=e\right\}\right.$ at an exponential rate.

Proof: Let $\omega$ be a positive number. The closed-loop equations of motion can be written as

$$
\begin{aligned}
\frac{d}{d t}\left[\begin{array}{l}
x_{1} \\
x_{2}
\end{array}\right] & =\left[\begin{array}{cc}
\beta\left(e-x_{3}\right) & -\omega \\
\omega & \beta\left(e-x_{3}\right)
\end{array}\right]\left[\begin{array}{l}
x_{1} \\
x_{2}
\end{array}\right] \\
\dot{x}_{3} & =\omega\left(x_{1}^{2}+x_{2}^{2}\right)-\alpha x_{3} .
\end{aligned}
$$

Introduce $\rho=\ln \left(x_{1}^{2}+x_{2}^{2}\right)$ and observe that $\rho$ and $x_{3}$ satisfy

$$
\begin{aligned}
\dot{\rho} & =2 \beta\left(e-x_{3}\right) \\
\dot{x}_{3} & =-\alpha x_{3}+\omega e^{\rho} .
\end{aligned}
$$

Eliminating $x_{3}$ gives

$$
\ddot{\rho}+\alpha \dot{\rho}+2 \beta \omega e^{\rho}=2 \alpha \beta e .
$$

This equation has a single equilibrium point, $\rho_{0}=\ln (\alpha e / \omega)$. It corresponds to $x_{3}=e$. The derivative of $v(\dot{\rho}, \rho)=(1 / 2) \dot{\rho}^{2}+$ 


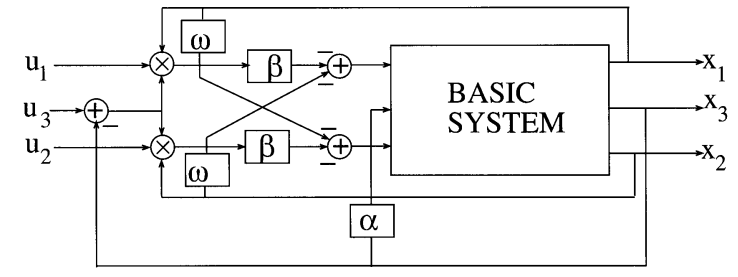

Fig. 2. Block diagram of the feedback controller of Theorem 1.

$2 \beta \omega e^{\rho}-2 \alpha \beta e \rho$ along solutions of the differential equation is $-\alpha \dot{\rho}^{2}$ and thus the equilibrium solution of the equation for $\rho$ is asymptotically stable. Observe that $x_{3}=e-\dot{\rho} / 2 \beta$ and so as $t$ approaches infinity $\left(\rho, x_{3}\right)$ approaches $(\ln \alpha e / \omega, e)$ from all initial values $\left[\rho(0), x_{3}(0)\right]$. However, $\left(x_{1}, x_{2}\right)=(0,0)$ does not correspond to a finite value of $\rho$ and, thus, it is excluded from this analysis; see Remark 1 . This concludes the proof.

A block diagram for this system is shown in Fig. 2. Linearizing the equation for $\rho$ about the equilibrium solution $\rho=$ $\ln (\alpha e / \omega)$ gives

$$
\ddot{\delta}+\alpha \dot{\delta}+2 \alpha \beta e \delta=0
$$

The eigenvalues of this second-order equation are

$$
s_{i}=\frac{\alpha}{2}\left(-1 \pm \sqrt{1-8 \frac{\beta e}{\alpha}}\right) .
$$

The system is critically damped when $4 \beta e=\alpha$.

Remark 1: If the Lyapunov function used in this proof is expressed in terms of $x$ instead of $\rho$, it takes the form

$$
v_{1}(x)=2 \beta^{2}\left(e-x_{3}\right)^{2}+2 \beta \omega\left(x_{1}^{2}+x_{2}^{2}\right)-\alpha \beta e \ln \left(x_{1}^{2}+x_{2}^{2}\right)
$$

which takes on its minimum value at $x_{1}^{2}+x_{2}^{2}=\alpha e / \omega, x_{3}=e$. The derivative is $\dot{v}_{1}=-4 \alpha \beta^{2}\left(x_{3}-e\right)^{2}$. If $e=0$, this function is well defined even for $x_{1}^{2}+x_{2}^{2}=0$. Thus, we observe that the null solution of the system defined in the theorem statement is asymptotically stable in the large for all $e \geq 0$. This slight extension of Theorem 1 will play a role in the later developments.

As mentioned before, in some applications the feedback control law is selected so that an increase in the load causes an increase in both the amplitude and the frequency of the oscillation. The following generalization of Theorem 1 incorporates this effect. It makes use of the control laws $u_{1}=-\left(1+k\left(x_{1}^{2}+\right.\right.$ $\left.x_{2}^{2}\right) \omega x_{2}+\beta\left(e-x_{3}\right) x_{1}$ and $u_{2}=-\left(1+k\left(x_{1}^{2}+x_{2}^{2}\right)\right) \omega x_{1}+$ $\beta\left(e-x_{3}\right) x_{2}$.

Theorem 2: If $x$ and $u$ are related by

$$
\frac{d}{d t}\left[\begin{array}{l}
x_{1} \\
x_{2} \\
x_{3}
\end{array}\right]=\left[\begin{array}{c}
u_{1} \\
u_{2} \\
x_{1} u_{2}-x_{2} u_{1}-\alpha x_{3}
\end{array}\right]
$$

with $\alpha>0$, then there exists $\omega \in \mathbb{R}$ such that for any $\beta, k>0$ and any $e>0$ the control law

$$
\left[\begin{array}{l}
u_{1} \\
u_{2}
\end{array}\right]=\left[\begin{array}{c}
-s(x) \omega x_{2}+\beta\left(e-x_{3}\right) x_{1} \\
s(x) \omega x_{1}+\beta\left(e-x_{3}\right) x_{2}
\end{array}\right]
$$

with $s(x)=\left(1+k x_{1}^{2}+x_{2}^{2}\right)$ defines a closed-loop system that admits a one-parameter family of periodic solutions of the form

$$
x_{p}(t)=\left[\begin{array}{c}
m \cos \left(\omega_{0} t+\phi\right) \\
m \sin \left(\omega_{0} t+\phi\right) \\
e
\end{array}\right], \quad 0 \leq \phi<2 \pi
$$

with

$$
m^{2}=\frac{1}{2 k}\left(-1+\sqrt{1+\frac{4 \alpha e k}{\omega}}\right)
$$

and

$$
\omega_{0}=\frac{\omega}{2}\left(1+\sqrt{1+\frac{4 \alpha e k}{\omega}}\right) .
$$

Each such periodic solution is stable and all solutions starting off the line $x_{1}=x_{2}=0$ approach the circle defined by $M_{\infty}=$ $\left\{\left(x_{1}, x_{2}, x_{3}\right) \mid\left(x_{1}^{2}+x_{2}^{2}=\alpha e / \omega_{0} ; x_{3}=e\right\}\right.$ at an exponential rate.

Proof: Let $\omega$ be any positive number. The closed-loop equations of motion are

$$
\begin{aligned}
\frac{d}{d t}\left[\begin{array}{l}
x_{1} \\
x_{2}
\end{array}\right] & =\left[\begin{array}{cc}
\beta\left(e-x_{3}\right) & -s(x) \omega \\
s(x) \omega & \beta\left(e-x_{3}\right)
\end{array}\right]\left[\begin{array}{l}
x_{1} \\
x_{2}
\end{array}\right] \\
\dot{x}_{3} & =s(x) \omega\left(x_{1}^{2}+x_{2}^{2}\right)-\alpha x_{3} .
\end{aligned}
$$

As before, introduce $\rho=\ln \left(x_{1}^{2}+x_{2}^{2}\right)$ and observe that

$$
\begin{aligned}
\dot{\rho} & =2 \beta\left(e-x_{3}\right) \\
\dot{x}_{3} & =-\alpha x_{3}+\left(1+k e^{\rho}\right) \omega e^{\rho} .
\end{aligned}
$$

In this case, the elimination of $x_{3}$ yields the equation

$$
\ddot{\rho}+\alpha \dot{\rho}+2 \beta \omega\left(e^{\rho}+k e^{2 \rho}\right)=2 \alpha \beta e .
$$

The equation $\omega\left(e^{\rho}+k e^{2 \rho}\right)=\alpha e$ is quadratic in the unknown $e^{\rho}$. Solving it for a positive root, we see that the equilibrium condition is

$$
e^{\rho_{0}}=\frac{-1}{2 k}+\frac{1}{2 k} \sqrt{1+\frac{4 \alpha k e}{\omega}}
$$

corresponding to $x_{3}=e$. The derivative of the Lyapunov function

$$
\begin{aligned}
v(\dot{\rho}, \rho) & =\frac{1}{2} \dot{\rho}^{2}+\int_{\rho_{0}}^{\rho} 2 \beta \omega\left(e^{\mu}+k e^{2 \mu}\right)-2 \alpha \beta e d \mu \\
& =\frac{1}{2} \dot{\rho}^{2}+\left.\left(2 \beta \omega e^{\mu}+\beta \omega k e^{2 \mu}-2 \alpha \beta e \mu\right)\right|_{\rho_{0}} ^{\rho}
\end{aligned}
$$

along solutions of this equation is $\dot{v}=-\alpha \dot{\rho}^{2}$ and thus the equilibrium solution $\rho=\rho_{0}$ is asymptotically stable. Linearizing the equation for $\rho$ about the equilibrium point gives

$$
\ddot{\delta}+\alpha \dot{\delta}+\beta\left(4 \alpha e+\frac{\omega}{k}-\sqrt{\frac{\omega^{2}}{k^{2}}+\frac{4 \alpha e \omega}{k}}\right) \delta=0 .
$$




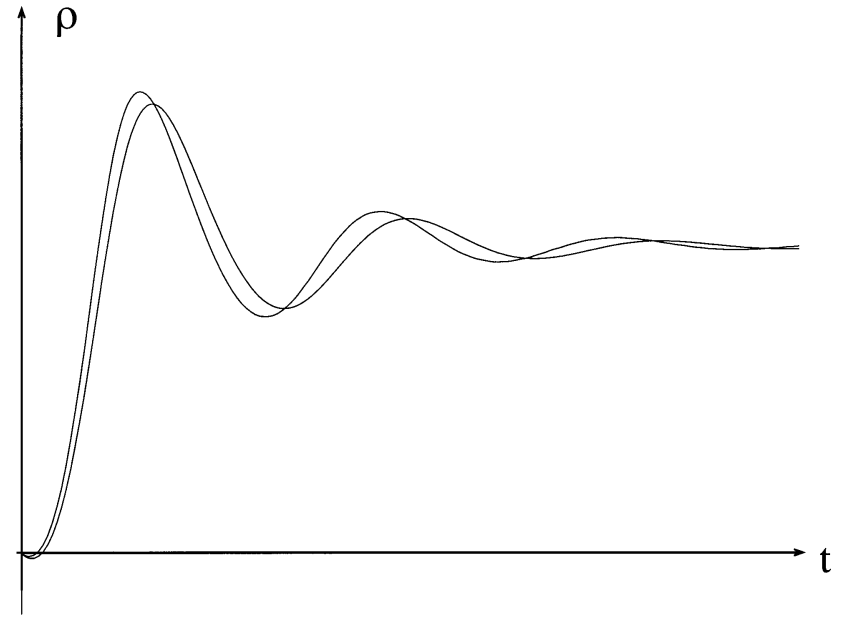

Fig. 3. Sample transient responses of $x_{3}$ with $\mathrm{k}=0$ (no frequency adjustment) and $\mathrm{k}=1$ (frequency adjustment). The system with the frequency adjustment term produces the faster response.

The Taylor series expansion about $k=0$ of the the coefficient of $\delta$ is

$4 \alpha \beta e+\frac{\beta \omega}{k}\left(1-\sqrt{1+\frac{4 \alpha e k}{\omega}}\right)=2 \alpha \beta e+\frac{2 \alpha^{2} \beta e^{2} k}{\omega}+\ldots$

If, for $k=0$, the roots are complex then the effect of a small increase in $k$ is to increase the resonant frequency, thus increasing the speed of response as shown in Fig. 3.

Remark 2: The particular choice of the frequency adjustment term $s(x)$ in Theorem 2 is not significant. Work in the biological literature suggests that a function that grows linearly with amplitude, such as $s(x)=1+k \sqrt{x_{1}^{2}+x_{2}^{2}}$ might better fit experimental data. The use of $s(x)=1+k \sqrt{x_{1}^{2}+x_{2}^{2}}$ in place of $s(x)=1+k\left(x_{1}^{2}+x_{2}^{2}\right)$ results in a cubic equation for $m^{2}$ but does not change the qualitative properties of our analysis.

\section{HIGHER DIMENSIONAL SYSTEMS}

Although the models of the previous section provide useful insight about how a system operates, more detailed models containing additional degrees of freedom, e.g., those needed to describe both the piston and the valve motion for an internal combustion engine, may be needed. For this reason, we now consider the extension of the previous results to higher dimensional problems. This involves significant new aspects because $X$ now typically takes on values in a space whose dimension is higher than the dimension of $u$ and it is only possible to achieve perfect regulation on a lower dimensional subspace. As it happens, it is possible to achieve perfect regulation in certain subspaces while ensuring that the average value is zero on a complementary subspace. The model will be the $m+m(m-1) / 2$-dimensional system introduced before

$$
\dot{x}=u \quad \dot{X}=x u^{T}-u x^{T}+U
$$

with $U=-\alpha X$.

Notation: Let $\operatorname{Skew}(m)$ denote the vector space of real, $m$ by $m$ skew-symmetric matrices. We regard this as an inner product space with $\left\langle X_{1}, X_{2}\right\rangle=\operatorname{tr}\left(\mathrm{X}_{1}^{\mathrm{T}} \mathrm{X}_{2}\right)$. If $\mathbb{F}$ is a subspace of Skew $(m)$ then $\pi_{\mathbb{F}}$ denotes the orthogonal prjection which projects $\operatorname{Skew}(m)$ onto $\mathbb{F}$. If $m$ is even, $\operatorname{Skew}(m)$ has a family of $(m / 2)$-dimensional subspaces with the property that any two elements in one of these subspaces commute. There are no subspaces of dimension greater than $m / 2$ that have this property. One such maximal commuting set is given by

$$
\mathbb{H}=\left\{H \mid H=\left[\begin{array}{cccc}
h_{1} J & 0 & \ldots & 0 \\
0 & h_{2} J & \ldots & 0 \\
\ldots & \ldots & \ldots & \ldots \\
0 & 0 & \ldots & h_{\frac{m}{2}} J
\end{array}\right] \quad h_{i} \in \mathbb{R}\right\}
$$

with $J$ being

$$
J=\left[\begin{array}{cc}
0 & 1 \\
-1 & 0
\end{array}\right]
$$

If $m$ is odd the maximal commuting subalgebra is of dimension $(m-1) / 2$ and the given choice of $\mathbb{H}$ must be modified by adding a one dimension 0 block at the lower right. This is one of many such facts playing a role in the classification of simple Lie algebras. Reference [12] is a readable account and [13] describes a number of applications of these ideas in control. In the literature on Lie algebras, maximal commuting subalgebras are called Cartan subalgebras. Cartan subalgebras are not unique

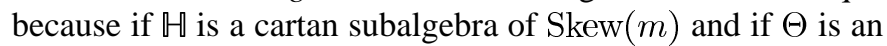
orthogonal matrix, then $\Theta \leftrightarrow \Theta^{T}$ is also a Cartan subalgebra. In fact, all Cartan subalgebras of $\operatorname{Skew}(m)$ can be generated from the particular block diagonal one given above using this relationship. Adopting this language, we may say that Theorem 3 gives conditions under which it is possible to achieve perfect regulation of $X$ on a Cartan subalgebra while forcing $X$ to have a zero average value on its orthogonal complement.

Finally, we introduce one nonstandard definition isolating an idea that plays an important role here. By a $d$-dimensional basic cone in $\mathbb{H}$ we understand any subset of $\mathbb{H}$ consisting of all elements expressible as

$$
E=\sum_{i=1}^{d} \alpha_{i} E_{i}, \quad \alpha_{i}>0
$$

with the $E_{i} \in \mathbb{H}$ being linearly independent and of rank two. Notice that the definition requires that the $\alpha_{i}$ be strictly positive.

We will say that a function $f(\cdot)$ taking on values in $\mathbb{R}^{n}$ is quasi-periodic if there exists a $n$ by $r$ constant matrix $C$, a real $r$ by $r$ matrix $\Omega=-\Omega^{T}$ and a $r$-dimensional vector $z$ such that $f(t)=C e^{\Omega t} z$. Such functions will be periodic if there exists a number $\omega_{0}$ such that all the eigenvalues of $\Omega$ are of the form $i r_{j} \omega_{0}$ with the $r_{j}$ being rational numbers.

Theorem 3: Suppose that $x(t), u(t) \in \mathbb{R}^{m}$ and $X(t) \in$ Skew $(m)$ are related by

$$
\dot{x}(t)=u(t) \quad \dot{X}(t)=x(t) u^{T}(t)-u(t) x^{T}(t)-\alpha X(t)
$$

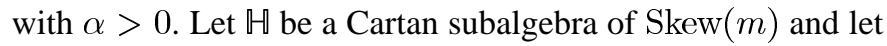
$\mathbb{E}$ be a $d$-dimensional basic cone in $\mathbb{H}$. Then, there exists $\Omega \in \mathbb{H}$ having $2 d$ distinct nonzero eigenvalues such that for any $\beta>0$ and any $E \in \mathbb{E}$, the control law

$$
u=\Omega x+\beta \Omega \pi_{\sharp}(E-X) x
$$


defines a closed-loop system which admits quasi-periodic solutions taking the form

$$
x_{p}(t)=e^{\Omega t} x_{0} \quad X_{p}(t)=E+\frac{d}{d t} e^{\Omega t} A e^{-\Omega t} \quad A=-A^{T}
$$

with $\pi_{\sharp}\left(X_{p}\right)=E$. These solutions take the form

$$
x_{p}(t)=e^{\Omega t} x_{0} \quad X_{p}(t)=E+\frac{d}{d t} e^{\Omega t} A e^{-\Omega t} \quad A=-A^{T}
$$

with $x_{0}$ a solution of $\pi_{H}\left(x_{0} x_{0}^{T} \Omega+\Omega x_{0} x_{0}^{T}\right)+E=0$. Moreover

1) each such solution is stable and if Span $\left\{x(0), \Omega x_{0}, \ldots \Omega^{m-1} x_{0}\right\}=\operatorname{Range}(E)$, then the corresponding trajectory approaches one such solution;

2) $\lim _{T \rightarrow \infty}(1 / T) \int_{0}^{T} X_{p}(t) d t=E$;

3) each such solution is periodic if the eigenvalues of $\Omega$ are rational multiples of a single number, $i \omega_{0}$.

Before proving the theorem, we give a few preliminary results.

Lemma 1: If $(x, X)$ satisfies

$$
\begin{aligned}
\dot{x} & =\Omega x+\beta \Omega \pi_{\nVdash}(E-X) x \\
\dot{X} & =-x x^{T} \Omega-\Omega x x^{T}+-\beta\left[x x^{T}, \Omega \pi_{\sharp}(E-X)\right]-\alpha X
\end{aligned}
$$

and if $\Theta$ is orthogonal, then $\left(y=\Theta x, Y=\Theta X \Theta^{T}\right)$ satisfies the same equation with $\Omega$ replaced by $\Theta \Omega \Theta^{T}, E$ replaced by $\Theta E \Theta^{T}$ and $\mathbb{H}$ replaced by $\Theta \Vdash \Theta^{T}$

Proof: This can be verified directly by differentiating $\Theta_{x}$ and $\Theta X \Theta^{T}$. The only point that is not immediate is to check that $\Theta\left(\pi_{\nVdash}(X)\right) \Theta^{T}=\left(\pi_{\Theta \mapsto \Theta^{T}}\left(\Theta X \Theta^{T}\right)\right)$, but this follows from the definitions.

Lemma 2: If $\Omega$ and $E$ are elements of $\operatorname{Skew}(m)$ then there exists unique skew-symmetric matrices $S$ and $F$ such that $E=$ $S+[\Omega, F]$ with $[S, \Omega]=0$ and $F$ orthogonal to every matrix commuting with $\Omega$.

Proof: The identity $\operatorname{tr}(A[B, C])=\operatorname{tr}([C, A] B)$ shows that the range of the operator $\operatorname{ad}_{\Omega}(\cdot)=[\Omega . \cdot]$ is the orthogonal complement of its null space.

Lemma 3: Let $\mathcal{C}(\Omega)$ denote the set of all matrices commuting with $\Omega$. Suppose $\Omega, \bar{\Omega} \in \operatorname{Skew}(m)$. Then

$$
\int_{0}^{t} e^{\Omega \tau} \bar{\Omega} e^{-\Omega \tau} d \tau=\pi_{\mathcal{C}(\Omega)}(\bar{\Omega}) t+P(t)
$$

where $P(t)$ is quasi-periodic.

Proof: Express $E$ as in Lemma 2, $E=S+[\Omega, F]$. Now, observe that

$$
\frac{d}{d \tau} e^{\Omega \tau} F e^{-\Omega \tau}=e^{\Omega \tau}[\Omega, F] e^{-\Omega \tau}
$$

Thus

$$
\begin{aligned}
\int_{0}^{t} e^{\Omega \tau} E e^{-\Omega \tau} d \tau & =\int_{0}^{t} e^{\Omega \tau} S e^{-\Omega \tau}+\frac{d}{d t}\left(e^{\Omega \tau} F e^{-\Omega t}\right) d \tau \\
& =S t+e^{\Omega \tau} F e^{-\Omega t}-F .
\end{aligned}
$$

Clearly, the last two terms are quasi-periodic.
Lemma 4: Let $\mathbb{H}$ be a Cartan subalgebra of $\operatorname{Skew}(m)$ and let $\mathbb{E}$ be a basic cone of dimension $d$ in $\mathbb{H}$. Then, there exists $\Omega \in \mathbb{H}$ such that for each $E$ in $\mathbb{E}$ we can find $x \in \mathbb{R}^{m}$ satisfying

$$
\pi_{\nVdash}\left(x x^{T} \Omega+\Omega x x^{T}\right)+E=0 .
$$

Moreover, if $\Omega$ is such that there exists some $x$ providing this representation then there is a $d+\nu$-parameter family of $x$ 's satisfying this equation where $\nu$ is the dimension of the kernel of $\Omega$.

Proof: We begin by showing that the claim is true if $E$ is $2 \times 2$. In this case, $\mathbb{H}=\operatorname{Skew}(2)$ and the projection $\pi_{\mathbb{H}}$ has no effect. We only need to show that there exists an $\Omega$ such that it is possible to solve

$$
\begin{aligned}
& {\left[\begin{array}{ll}
x_{1} x_{1} & x_{1} x_{2} \\
x_{1} x_{2} & x_{2} x_{2}
\end{array}\right]\left[\begin{array}{cc}
0 & \omega_{1} \\
-\omega_{1} & 0
\end{array}\right]} \\
& \quad+\left[\begin{array}{cc}
0 & \omega_{1} \\
-\omega_{1} & 0
\end{array}\right]\left[\begin{array}{ll}
x_{1} x_{1} & x_{1} x_{2} \\
x_{1} x_{2} & x_{2} x_{2}
\end{array}\right]+\left[\begin{array}{cc}
0 & -e_{1} \\
e_{1} & 0
\end{array}\right]=0
\end{aligned}
$$

for each $E$ in the basic cone $\mathbb{E}$. There are essentially two possibilities. Either $\mathbb{E}$ consists of the set of two-by-two skew-symmetric matrices whose 12-element is positive or else it consists of the set of $2 \times 2$ skew-symmetric matrices whose 12-element is negative. Let $\Omega$ be any skew-symmetric matrix that is a negative multiple of an $E \in \mathbb{E}$. Then, $e_{1} / \omega_{1}$ is negative and there is a one-parameter family of solutions of

$$
x_{1}^{2}+x_{2}^{2}-\frac{e_{1}}{\omega_{1}}=0 .
$$

This establishes the lemma in the case that $m=2$. Now consider the general case. As noted above, there exists an orthogonal transformation that puts $\mathbb{H}$ in block diagonal form. Thus, the basic cone has a block diagonal set of generators. We can order the basis of $\mathbb{H}$ such that the generators of $\mathbb{E}$ occur in the upper left $2 d$-by- $2 d$ block of Skew $(m)$. We take the upper-left $2 d$-by- $2 d$ block of $\Omega$ to be a negative multiple of some element of $\mathbb{E}$ and let the remaining elements be arbitrary subject to the constraint $\Omega \in \mathbb{H}$. To find $x$, we need only work one block at a time, consistent with the solution found in the two-by-two case. Of course, this will result in $x x^{T} \Omega+\Omega x x^{T}$ being nonzero off the diagonal but the projection $\pi_{\sharp}$ will annihilate the off-diagonal elements and result in a solution of $\pi_{\boxplus}\left(x x^{T} \Omega+\Omega x x^{T}\right)+E=0$. If $\Omega$ is invertable this solution is unique to within choices previously described. However if $\Omega$ has a $\nu$-dimensional kernel then $x$ is arbitrary in the kernel, leading to the formula in the lemma.

Proof of Theorem 3: Using freely the commutivity implied by the hypothesis we can write the closed-loop equations of motion as

$$
\begin{aligned}
& \dot{x}=\Omega x+\beta \Omega \pi_{\mathfrak{H}}(E-X) x \\
& \dot{X}=-x x^{T} \Omega-\Omega x x^{T}+-\beta\left[x x^{T}, \Omega \pi_{\sharp}(E-X)\right]-\alpha X \text {. }
\end{aligned}
$$

Assuming $m$ is even, we can find an orthogonal matrix $\Theta$ such that after the change of coordinates $x \mapsto \Theta x ; X \mapsto \Theta X \Theta^{T}, \mathbb{H}$ takes the form

$$
\mathbb{H}=\left\{H \mid H=\left[\begin{array}{cccc}
h_{1} J & 0 & \ldots & 0 \\
0 & h_{2} J & \ldots & 0 \\
\ldots & \ldots & \ldots & \ldots \\
0 & 0 & \ldots & h_{\frac{m}{2}} J
\end{array}\right] \quad h_{i} \in \mathbb{R}\right\}
$$


as discussed in the definition of the Cartan subalgebra and in Lemma 1 . We can order the basis such that when $E$ is expressed in the same coordinate system it is nonzero only in the upper left $2 d \times 2 d$ block.

These equations have an important property that can be expressed as

$$
\pi_{\mathbb{H}}\left(\left[x x^{T}, \Omega \pi_{\mathbb{H}}(E-X)\right]\right)=0 .
$$

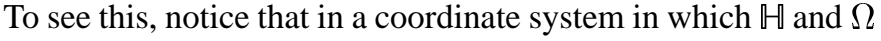
are block diagonal, $\Omega \pi_{\mathfrak{H}}(X-E)$ is block diagonal with each two-by-two block being a multiple of the identity. Thus, if we let $X_{\pi}=\pi_{\mathbb{R}}(X)$ then

$$
\begin{aligned}
\dot{x} & =\Omega x+\beta \Omega\left(E-X_{\pi}\right) x \\
\dot{X}_{\pi} & =-\pi_{H}\left(x x^{T} \Omega+\Omega x x^{T}\right)-\alpha X_{\pi} .
\end{aligned}
$$

The subsystem consisting of the upper $2 d$ equations can be arranged as a set of decoupled equations, each $2 \times 2$ block taking the form

$$
\begin{aligned}
\frac{d}{d t}\left[\begin{array}{l}
a \\
b
\end{array}\right] & =\left[\begin{array}{cc}
\beta \omega(e-c) & -\omega \\
\omega & \beta \omega(e-c)
\end{array}\right]\left[\begin{array}{l}
a \\
b
\end{array}\right] \\
\dot{c} & =\omega\left(a^{2}+b^{2}\right)-\alpha c .
\end{aligned}
$$

As in the proof of Theorem 1, introduce new variables $\rho_{i}=$ $\ln \left(a_{i}^{2}+b_{i}^{2}\right)$ and observe that $\rho_{i}$ and $c_{i}$ satisfy

$$
\begin{aligned}
& \dot{\rho}_{i}=2 \beta \omega_{i}\left(e_{i}-c_{i}\right) \\
& \dot{c}_{i}=-\alpha c_{i}+\omega e^{\rho_{i}} .
\end{aligned}
$$

Differentiating the first equation and using the second to eliminate $c_{i}$, we get

$$
\ddot{\rho}_{i}+\alpha \dot{\rho}_{i}+2 \beta \omega_{i}^{2} e^{\rho_{i}}=2 \alpha \beta \omega_{i} e_{i} .
$$

The properties claimed for $x$ and $\pi_{\mathbb{H}}(X)$ now follow from the analysis done in the proof of Theorem 1. (What is called $\beta$ there is now to be identified with $\omega_{i} \beta$.) Note that the equations in $\rho$ are asymptotically stable but the equations in $x$ are only neutrally stable because of the neutral stability of the relative phases.

To complete the proof in the case $m$ is even we need to use Lemma 3 to establish the appropriate properties for the projection of $X$ onto the complement of $\mathbb{H}$. If the eigenvalues of $\Omega$ are unrepeated then $\mathcal{C}(\Omega)=\mathbb{H}$ and lemma three applies directly. On the other hand, if $2 d<m$ then the eigenvalues may be repeated. As noted in the proof of Lemma 4, if the eigenvalues of $\Omega$ are all nonzero the solutions of $\pi_{\mathbb{H}}\left(x x_{0}^{T} \Omega+\Omega x_{0} x_{0}^{T}\right)+$ $E=0$ will be zero except for the first $2 d$ entries. This implies that $X_{p}$ will be zero outside the upper left $2 d$-by- $2 d$ block and Lemma three implies that $X_{p}$ has the desired properties within the $2 d$-by- $2 d$ block. The fact that $X_{p}$ is quasi-periodic is established by Lemma 3 together with the remark that the input-output stable linear system $\dot{X}=-X+U(t)$ will have a unique quasi-periodic solution for each quasi-periodic $U$. If $x(0)$ is a cyclic vector for $\Omega$ then none of the blocks can have a zero initial condition vector, necessary for the application of Theorem 1. Thus there exists stable solutions $\left(x_{p}, X_{p}\right)$ with the given properties defining a quasi-periodic solution. If the eigenvalues of $\Omega$ are rational multiples of a single imaginary number then $e^{\Omega_{p} t}$ is periodic and hence the solution is periodic and not just quasi-periodic.

Finally, if $m$ is odd the equations can be arranged as a set of $(m-1) / 2$ blocks in the form just discussed but now with a final equation which necessarily takes the form $\dot{x}_{m}=0$. The variable $x_{m}$ does not enter the other equations for $x$ and is neutrally stable as required by the theorem statement. It enters the equation for $\dot{X}$ but because $x_{m}$ is a constant and multiplies only functions with average value zero, it does not affect the conclusions reached above.

Example 1: Let $\nVdash$ be the set of $4 \times 4$ block diagonal matrices and let $\mathbb{E}$ be the cone consisting of those elements of $\mathbb{H}$ that have negative entries in the 12-element and the 34-element. Let $E \in \mathbb{H}$ be given by

$$
E=\left[\begin{array}{cccc}
0 & -3 & 0 & 0 \\
3 & 0 & 0 & 0 \\
0 & 0 & 0 & -2 \\
0 & 0 & 2 & 0
\end{array}\right]
$$

and let $\Omega \in \mathbb{H}$ be

$$
\Omega=\left[\begin{array}{cccc}
0 & 1 & 0 & 0 \\
-1 & 0 & 0 & 0 \\
0 & 0 & 0 & 2 \\
0 & 0 & -2 & 0
\end{array}\right]
$$

The theorem asserts that there is a two parameter family of $x$ 's satisfying $x x^{T} \Omega+\Omega x x^{T}+E=0$. The corresponding two parameter family of solutions can be expressed as

$$
x(t)=\left[\begin{array}{c}
\sqrt{3} \cos (t+\theta) \\
\sqrt{3} \sin (t+\theta) \\
\cos (2 t+\phi) \\
\sin (2 t+\phi)
\end{array}\right]
$$

In biological systems, synchronization might relate to maintaining a particular phase relationship between the action of muscle groups used for breathing. In an electrical power grid, synchronization might be necessary to coordinate the frequency and phase of various generators. Clearly, the maintenance of correct phase relationships can be of critical importance. Of course synchronization is only meaningful in cases where $\Omega$ has repeated eigenvalues. If part of the goal of the feedback control system is to synchronize various modes, it is essential that we choose $\Omega$ appropriately. Theorem 3 allows repeated eigenvalues in $\Omega$ only when the cone $\mathbb{E}$ is of dimension less than $m-1$. The following example illustrates the simultaneous regulation of amplitude and the relative phase of two modes.

Example 2: Let $m=4$ and let $\mathbb{H}$ be the linear span of the two commuting matrices

$$
H_{1}=\left[\begin{array}{cccc}
0 & -1 & 0 & 0 \\
1 & 0 & 0 & 0 \\
0 & 0 & 0 & -1 \\
0 & 0 & 1 & 0
\end{array}\right] \quad H_{2}=\left[\begin{array}{cccc}
0 & 1 & 0 & 1 \\
-1 & 0 & -1 & 0 \\
0 & 1 & 0 & 1 \\
-1 & 0 & -1 & 0
\end{array}\right] .
$$

Let $\mathbb{E}$ be the positive multiples of $\mathrm{H}_{2}$. Because the elements of $\mathbb{E}$ are only of rank two, we can limit $\Omega$ to one pair of distict eigenvalues. Let $\Omega=\omega H_{1}$. Theorem 3 asserts that there is only a one parameter family of $x$ 's satisfying $x x^{T} \Omega+\Omega x x^{T}+E=0$ and that there is a periodic solution of the feedback equations. 
The corresponding one-parameter family of periodic solutions is

$$
x(t)=\frac{1}{\omega}\left[\begin{array}{c}
\cos (\omega t+\theta) \\
\sin (\omega t+\theta \\
\cos (\omega t+\theta) \\
\sin (\omega t+\theta)
\end{array}\right]
$$

so that $x_{1}$ and $x_{3}$ are necessarily synchronized.

As might be expected, there is an adjustable frequency version of Theorem 3 that stands in relationship to Theorem 3 as Theorem 2 stands to Theorem 1. In the theorem statement we use $\Omega^{\dagger}$ to denote the Moore-Penrose inverse.

Theorem 4: Suppose that $x(t), u(t) \in \mathbb{R}^{m}$ and $X(t) \in$ Skew $(m)$ are related by

$$
\dot{x}(t)=u(t) \quad \dot{X}(t)=x(t) u^{T}(t)-u(t) x^{T}(t)-\alpha X(t)
$$

with $\alpha>0$. Let $\mathbb{H}$ be a Cartan subalgebra of $\operatorname{Skew}(m)$ and let $\mathbb{E}$ be a $d$-dimensional basic cone in $\mathbb{H}$. Then there exists $\Omega \in \mathbb{H}$ having $2 d$ distinct nonzero eigenvalues such that for any $\beta, k>$ 0 and any $E \in \mathbb{E}$, the control law

$$
u=s(x) \Omega x+\beta \Omega \pi_{\mathbb{H}}(E-X) x \quad U=-\alpha X
$$

with $s(x)=\left(1+k x^{T} x\right)$, defines a closed-loop system which admits a $d$-parameter family of quasi-periodic solutions $\left(x_{p}\right.$, $\left.X_{p}\right)$,

$$
x_{p}(t)=e^{\Omega_{0} t} x_{0} \quad X_{p}(t)=E+\frac{d}{d t} e^{\Omega_{0} t} A e^{-\Omega_{0} t} \quad A=-A^{T}
$$

with

$$
\Omega_{0}=\left(\frac{1}{2}+\frac{1}{2} \sqrt{1+4 \alpha k \operatorname{tr}\left(\Omega^{\dagger} \mathrm{E}\right)}\right) \Omega
$$

and $\pi_{\mathcal{C}(\Omega)}\left(x_{0} x_{0}^{T} \Omega_{0}+\Omega_{0} x_{0} x_{0}^{T}\right)+E=0$. Moreover

1) each such solution stable;

2) $\lim _{T \rightarrow \infty}(1 / T) \int_{0}^{T} X_{p}(t) d t=E$;

3) each such solution is periodic if the eigenvalues of $\Omega$ are rational multiples of a single number, $i \omega_{0}$.

Proof: Note that because $\Omega$ and $\Omega_{0}$ are proportional, $\Omega_{0} \in$ $\mapsto$. The closed-loop equations of motion are

$$
\begin{aligned}
\dot{x}= & s(x) \Omega x+\beta \Omega \pi_{\mathbb{H}}(E-X) x \\
\dot{X}= & -s(x)\left(x x^{T} \Omega+\Omega x x^{T}\right) \\
& -\beta\left[x x^{T}, \Omega \pi_{\mathbb{H}}(E-X)\right]-\alpha X .
\end{aligned}
$$

Again, in view of Lemma 1, we may as well assume that both $E$ and $\Omega$ are in block diagonal form. In view of the properties of $\pi_{\sharp}$ we see that the equations for the diagonal blocks are decoupled from the off-diagonal terms but because of the $s(x)$ term, the diagonal blocks are coupled to each other. Initially, we consider the case where $m$ is even. The equations for the diagonal blocks take the form

$$
\begin{aligned}
\frac{d}{d t}\left[\begin{array}{c}
a_{i} \\
b_{i}
\end{array}\right] & =\left[\begin{array}{cc}
\beta\left(e_{i}-c_{i}\right) & -s(x) \omega_{i} \\
s(x) \omega_{i} & \beta\left(e_{i}-c_{i}\right)
\end{array}\right]\left[\begin{array}{c}
a_{i} \\
b_{i}
\end{array}\right] \\
\dot{c}_{i} & =\left(1+k x^{T} x\right) \omega\left(a_{i}^{2}+b_{i}^{2}\right)-\alpha c_{i} .
\end{aligned}
$$

Introduce $\rho_{i}=\ln \left(a_{i}^{2}+b_{i}^{2}\right)$ and observe that $\rho_{i}$ and $c_{i}$ satisfy

$$
\begin{aligned}
& \dot{\rho}_{i}=2 \beta\left(e_{i}-c_{i}\right) \\
& \dot{c}_{i}=-\alpha c_{i}+\left(1+k \sum_{j=1}^{\frac{m}{2}} e^{\rho_{j}}\right) \omega_{i} e^{\rho_{i}} .
\end{aligned}
$$

The first equation implies $\ddot{\rho}_{i}+\alpha \dot{\rho}_{i}=-\beta\left(\dot{c}_{i}+\alpha c_{i}\right)+2 \alpha \beta e_{i}$ and using the second to eliminate $c_{i}$, we get

$$
\ddot{\rho}_{i}+\alpha \dot{\rho}_{i}+2 \beta \omega_{i}\left(1+k \sum_{j=1}^{\frac{m}{2}} e^{\rho_{j}}\right) e^{\rho_{i}}=2 \alpha \beta e_{i} .
$$

Dividing by $\omega_{i}$ and then summing over $i$, we see that in equilibrium

$$
2 \beta\left(1+k \sum_{i=1}^{\frac{m}{2}} e^{\rho_{i}}\right) \sum_{i=1}^{\frac{m}{2}} e^{\rho_{i}}=2 \alpha \beta \sum_{i=1}^{\frac{m}{2}} \frac{e_{i}}{\omega_{i}} .
$$

Solving this quadratic equation for the sum implies that in equilibrium

$$
\sum_{i=1}^{\frac{m}{2}} e^{\rho_{i}}=\frac{-1}{2 k}+\frac{1}{2 k} \sqrt{1+4 \alpha k \sum_{i=1}^{\frac{m}{2}} \frac{e_{i}}{\omega_{i}}} .
$$

Having solved for the sum, the individual $\rho_{i}$ can be determined as

$$
e^{\rho_{i}}=\frac{\alpha e_{i}}{\omega_{i}}\left(1+k \sum_{i=1}^{\frac{m}{2}} e^{\rho_{i}}\right)^{-1}
$$

and, thus

$$
\left(1+k \sum_{j=1}^{\frac{m}{2}} e^{\rho_{i}}\right) \omega_{i}=\left(\frac{1}{2}+\frac{1}{2} \sqrt{1+4 \alpha k \sum_{j=1}^{\frac{m}{2}} \frac{e_{j}}{\omega_{j}}}\right) \omega_{i} .
$$

However

$$
\operatorname{tr}\left(\Omega^{-1} \mathrm{E}\right)=2 \sum_{\mathrm{j}=1}^{\frac{\mathrm{m}}{2}} \frac{\mathrm{e}_{\mathrm{j}}}{\omega_{j}}
$$

Notice that if the eigenvalues of $\Omega$ are unrepeated and $m$ is even, then $\Omega$ is necessarily invertible, $\Omega^{\dagger}=\Omega^{-1}$, and thus we have the expression for $\Omega_{0}$ given in the theorem statement.

The fact that the frequency adjustment term couples all the second-order oscillators together complicates the analysis. In order to prove local stability of this equilibrium solution, we linearize the equation for $\rho$ about the equilibrium solution $(\dot{\rho}, \rho)=$ $(0, \bar{\rho})$. This gives a set of coupled equations of the form

$$
\begin{array}{r}
{\left[\begin{array}{c}
\ddot{\delta}_{1} \\
\ddot{\delta}_{2} \\
\because . \\
\ddot{\delta}_{\frac{m}{2}}
\end{array}\right]+\left[\begin{array}{c}
\dot{\delta}_{1} \\
\dot{\delta}_{2} \\
\ldots \\
\dot{\delta}_{\frac{m}{2}}
\end{array}\right]+\left[\begin{array}{cccc}
2 e_{1}+q_{1} & q_{2} & \ldots & q_{m} \\
q_{1} & 2 e_{2}+q_{2} & \ldots & q_{\frac{m}{2}} \\
\ldots & \ldots & \ldots & \ldots \\
q_{1} & q_{2} & \ldots & 2 e_{\frac{m}{2}}+q_{\frac{m}{2}}
\end{array}\right]} \\
\\
\end{array}
$$


where $q_{i}=2 \omega_{i} k e^{\bar{\rho}_{i}}$. Abbreviate the coefficient of $\delta$ as $K$ and observe that we can express $K$ as $K=F Q$ where $Q=$ $\operatorname{diag}\left(2 q_{1} / e_{1}+1,2 q_{2} / e_{2}+1, \ldots, 2 q_{m / 2} / 2+1\right.$ and

$$
F=\left[\begin{array}{cccc}
\frac{2 e_{1}}{q_{1}}+1 & 1 & \ldots & 1 \\
1 & \frac{2 e_{2}}{q_{2}}+1 & \ldots & 1 \\
\ldots & \ldots & \ldots & \ldots \\
1 & 1 & \ldots & \frac{2 e_{m}}{q_{m}}+1
\end{array}\right]>0
$$

Writing

$$
F Q=\sqrt{Q}^{-1}(\sqrt{Q} F \sqrt{Q}) \sqrt{Q}
$$

we see that $F Q$ is similar to a positive-definite matrix. If we define $y$ as $y=\sqrt{Q}^{-1} x$ then $\ddot{y}+\dot{y}+\bar{F} y=0$ with $\bar{F}$ positive definite. Clearly, the null solution of this equation is asymptotically stable and so the equation for $\delta$ must be as well. Again, whereas the equations for $\rho$ imply asymptotically stability, the equations for $x$ imply only neutral stability because of the presence of relative phases.

\section{NONSINUSOIDAL PATTERNS}

Up until this point the explicit solutions we have developed have involved sinusoidal patterns. The feedback control laws used in Section IV results in a sinusoidal oscillation with area being generated at a constant rate. The constant rate generation property is of importance because it is this property that makes it possible for there to be a steady-state solution with $\pi_{\mathbb{H}}(X)$ constant. However, the examination of more detailed models often reveal considerations that make pure sinusoids less desirable than other wave shapes. There are other oscillatory patterns having the same key property of sweeping out "equal area in equal time."

Example 3: Consider the following modified version of the system treated in Theorem 1:

$$
\dot{x}_{1}=u_{1} \quad \dot{x}_{2}=u_{2} \quad \dot{x}_{3}=g\left(x_{1}\right) u_{2}-g\left(x_{2}\right) u_{1}+u_{3}
$$

with $g$ such that $x g(x) \geq 0$. consider a feedback control law of the form $u_{1}=-h\left(x_{2}\right) ; u_{2}=h\left(x_{1}\right)$, with $h$ being such that $h(x) x \geq 0$. If the equations

$$
\begin{aligned}
& \dot{x}_{1}=-h\left(x_{2}\right) \\
& \dot{x}_{2}=h\left(x_{1}\right) \\
& \dot{x}_{3}=-g\left(x_{1}\right) h\left(x_{1}\right)+g\left(x_{2}\right) h\left(x_{2}\right)-\alpha x_{3}
\end{aligned}
$$

are to admit a solution with $x_{3}$ constant, then along trajectories of $\dot{x}_{1}=-h\left(x_{2}\right)$ and $\dot{x}_{2}=h\left(x_{1}\right)$, the quantity $g\left(x_{1}\right) h\left(x_{1}\right)+$ $g\left(x_{2}\right) h\left(x_{2}\right)$ must be constant. If $H(x)=\int_{0}^{x} h(\mu) d \mu$, then it is easy to verify that the function

$$
v\left(x_{1}, x_{2}\right)=H\left(x_{1}\right)+H\left(x_{2}\right)
$$

is constant along solutions of $\dot{x}_{1}=-\omega h\left(x_{2}\right) ; \dot{x}_{2}=\omega h\left(x_{1}\right)$. Thus, if $g\left(x_{1}\right) h\left(x_{1}\right)+g\left(x_{2}\right) h\left(x_{2}\right)$ is constant on the constant value contours of $v$ we will have a system that admits solutions with $x_{3}$ constant.
We will say that $g$ and $h$ are matched if the aforementioned relationship holds. The condition for matching can be written more explicitly in terms of $h$ as

$$
h(x)=\frac{d H}{d x} \quad g(x)=\frac{\psi(H(x))}{h(x)}
$$

where $\psi$ is a monotone function such that $\psi(H(x)) / h(x)$ is everywhere defined.

Remark 3: Let $h(x)=x^{p}$ with $p$ an odd positive integer so that $H(x)=x^{p+1} /(p+1)$. Let $\psi(y)=a(p+1) y$ so that $g(x)=a x$ For large values of $p$ the corresponding oscillations will approach a square in the same sense that the level curves of $v\left(x_{1}, x_{2}\right)=x_{1}^{p+1}+x_{2}^{p+1}$ approach a square as $p$ becomes large. On the other hand, as $p$ approaches one, the level surfaces approach a diamond and $h$ approaches the signum function, $\operatorname{sgn}(x)$. Finally, we consider an example with $h$ and $g$ discontinuous. Let $h(x)=\operatorname{sgn}(x) e^{|x|}$ so that $H(x)=e^{|x|}-1$. If we let $\psi(H)=H+1$ then $g(x)=\operatorname{sgn}(x)$.

Building on these ideas, we now turn to the higher dimensional analogs. If $x$ is a vector and if $g$ is a map of the real line into itself, we adopt the notation

$$
g_{v}(x)=\left[\begin{array}{c}
g\left(x_{1}\right) \\
g\left(x_{2}\right) \\
\cdots \\
g\left(x_{m}\right)
\end{array}\right]
$$

Consider

$$
\dot{x}=u \quad \dot{X}=g_{v}(x) u^{T}-u g_{v}^{T}(x)+U .
$$

Rather than working with the energy function $(1 / 2)\|x\|^{2}+$ $(1 / 4)\|X\|^{2}$ as before, let

$$
v(x, X)=\sum H\left(x_{i}\right)+\frac{1}{4}\|X\|^{2}
$$

with $H$ being a nonnegative function that vanishes only when $x=0$. Let $h$ be the derivative of $H$. Observe that along solutions of the $g$-modified system

$$
\begin{aligned}
\frac{d}{d t} v & =\sum \frac{d H\left(x_{i}\right)}{d x_{i}} u_{i}+u^{T} X^{T} g_{v}(x) \\
& =\left\langle h_{v}(x)-X g_{v}, u\right\rangle+\frac{1}{2}\langle X, U\rangle
\end{aligned}
$$

the corresponding definition of $y$ such that the system is lossless is

$$
y=g_{v}(x)-X g_{v}(x) \quad Y=X .
$$

Setting $u$ equal to $\Omega y$ and $U=-\alpha X$ then gives

$$
\begin{aligned}
\dot{x} & =\Omega h_{v}(x)-\Omega X g_{v}(x) \\
\dot{X} & =-\left(g_{v}(x) h_{v}^{T}(x) \Omega+\Omega h_{v}(x) g_{v}^{T}(x)\right)-\alpha X .
\end{aligned}
$$

Theorem 5: Let $\alpha$ and $\beta$ be positive constants. Let $\Omega$ and $E$ be elements of $\operatorname{Skew}(m)$ such that $[E, \Omega]=0$ and $E \Omega>0$. Denote the set of all matrices commuting with $\Omega$ by $\mathbb{H}$. Suppose that $(x, X)$ and $(u, U)$ are related by

$$
\dot{x}=u \quad \dot{X}=g_{v}(x) u^{T}-u g_{v}^{T}(x)-\alpha X .
$$


Let $h=d H / d x$ be such that

$$
g(x) h(x)=\psi(H(x))
$$

for some function $\psi$. If the feedback control law

$$
u=\Omega h(x)+\beta \Omega \pi_{\nVdash}(E-X) g(x)
$$

defines a closed-loop system such that the resulting differential equations have piecewise differentiable solutions, then they admit a stable periodic solution on which $\pi_{\mathbb{\sharp}}(E-X)=0$.

Proof: The equations of motion for the closed-loop system are

$$
\begin{aligned}
\dot{x}= & \Omega h(x)+\beta \Omega \pi_{H}(E-X) g(x) \\
\dot{X}= & -g_{v}(x) h_{v}^{T}(x) \Omega-\Omega h_{v}(x) g_{v}^{T}(x) \\
& -\beta \pi_{H}\left(\left[g_{v}(x) g_{v}^{T}(x), \Omega(X-E)\right]\right)-\alpha X .
\end{aligned}
$$

As in the proof of Theorem 3, we observe that the bracket term is zero. Let $X_{\pi}=\pi_{\mathbb{H}}(X)$ and observe that

$$
\begin{aligned}
& \dot{x}=\Omega h_{v}(x)+\beta \Omega(X-E) g_{v}(x) \\
& \dot{X}_{\pi}=-\pi_{\sharp}\left(g_{v}(x) h_{v}^{T}(x) \Omega-\Omega h_{v}(x) g_{v}^{T}(x)\right)-\alpha X_{\pi} .
\end{aligned}
$$

This is just a set of decoupled equations of the type treated in the previous example.

\section{MUlTiPHASE SOLUTIONS}

Here, we indicate briefly how one can get a crude model for biped walking by joing together the methods of synchronization and oscillation shaping. We begin with an example illustrating a special form of oscillation shaping involving the signum function. Examples involving discontinuous functions offer a way of thinking about what is sometimes studied under the name piecewise holonomic systems [14]. We will build on this for the main example.

Example 4: Let $a$ be a positive constant. We consider specializing the results of the previous section by letting $h(x)=$ $\operatorname{sgn}(x) e^{|x|}$ and $g(x)=\operatorname{sgn}(x)$. If $x$ is two dimensional, this gives rise to

$$
\begin{aligned}
& \dot{x}_{1}=-\operatorname{sgn}\left(x_{2}\right) e^{\left|x_{2}\right|}+\left(a-x_{3}\right) \operatorname{sgn}\left(x_{1}\right) \\
& \dot{x}_{2}=\operatorname{sgn}\left(x_{1}\right) e^{\left|x_{1}\right|}+\left(a-x_{3}\right) \operatorname{sgn}\left(x_{2}\right) \\
& \dot{x}_{3}=e^{\left|x_{1}\right|}+e^{\left|x_{2}\right|}-x_{3} .
\end{aligned}
$$

It is not difficult to see that for each $\left(x_{1}(0), x_{2}(0)\right) \neq(0,0)$ there exists a piecewise smooth classical solution to these equations and as $t \rightarrow \infty$ solutions approach a periodic solution with $x_{1}$ and $x_{2}$ lying on a level set of the function $v\left(x_{1}, x_{2}\right)=$ $e^{\left|x_{1}\right|}+e^{\left|x_{2}\right|}$

Example 5: A simple and purely kinematic model for twolegged walking serves to illustrate a number of the concepts introduced in this paper. Suppose that $x$ is a point in $\mathbb{R}^{5}$ and that the equations of motion for $x$ are

$$
\begin{aligned}
& \dot{x}_{i}=u_{i} \quad 1 \leq i \leq 4 \\
& \dot{x}_{5}=\left(1-\operatorname{sgn}\left(x_{1}\right)\right) u_{2}+\left(1+\operatorname{sgn}\left(x_{3}\right)\right) u_{4} .
\end{aligned}
$$

The interpretation is that $x_{1}$ represents the vertical coordinate of one foot, say the right foot, and $x_{3}$ represents the vertical coor-

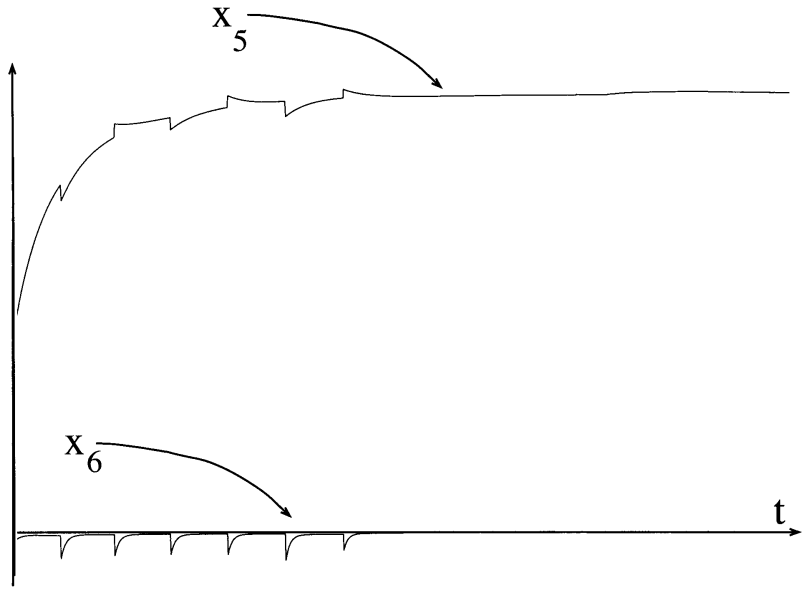

Fig. 4. Simulation of the biped showing the variables $x_{5}$ and $x_{6}$.

dinate of the left foot. When $x_{1}$ is positive, the right foot is not in contact with the ground and thus has no role in maintaining forward motion but that when it is zero it does. Similar remarks apply to $x_{3}$. We let $x_{2}$ denote the horizontal position of the right foot, measured relative to the center of mass of the body and let $x_{4}$ denote the horizontal position of the left foot relative to the center of mass of the body. Now, let $x_{5}$ represent the horizontal velocity of the center of mass of the body, relative to a fixed point. The idea is that when the right foot is touching the ground $\left(x_{1}=0\right)$ then the advance of $x_{5}$ is coupled to that of $u_{2}$, but not otherwise. Similar remarks apply to $x_{3}$ and $u_{4}$. Clearly, $x_{5}$ can progress at a uniform rate only if the $\left(x_{1}, x_{2}\right)$ subsystem is $\pi$ radians out of phase with the $\left(x_{3}, x_{4}\right)$ system. We add an additional state variable to the system, one that measures the phase angle between $x_{1}$ and $x_{3}$ using $\dot{x}_{6}=x_{1} u_{3}-x_{3} u_{1}$. If in steady state $x_{6}=0$, then the $x_{1}$ and $x_{3}$ are completely out of phase as desired. Consider then, an augmented set of equations

$$
\begin{aligned}
& \dot{x}_{1}=-\omega \operatorname{sgn}\left(x_{2}\right)+\beta\left(e-x_{5}\right) \operatorname{sgn}\left(x_{1}\right)+x_{6} \operatorname{sgn}\left(x_{2}\right) \\
& \dot{x}_{2}=\omega \operatorname{sgn}\left(x_{1}\right)+\left(e-x_{5}\right) \operatorname{sgn}\left(x_{2}\right)-x_{6} \operatorname{sgn}\left(x_{1}\right) \\
& \dot{x}_{3}=-\omega \operatorname{sgn}\left(x_{4}\right)+\left(e-x_{5}\right) \operatorname{sgn}\left(x_{3}\right)-x_{6} \operatorname{sgn}\left(x_{4}\right) \\
& \dot{x}_{4}=\omega \operatorname{sgn}\left(x_{3}\right)+\left(e-x_{5}\right) \operatorname{sgn}\left(x_{4}\right)+x_{6} \operatorname{sgn}\left(x_{3}\right) \\
& \dot{x}_{5}=\left(1+\operatorname{sgn}\left(x_{2}\right)\right) u_{1}+\left(1+\operatorname{sgn}\left(x_{3}\right)\right) u_{4}-x_{5} \\
& \dot{x}_{6}=x_{1} \dot{x}_{3}-x_{3} \dot{x}_{1}-\alpha x_{6}
\end{aligned}
$$

with $\omega=\omega_{0}+k\left(\left|x_{1}\right|+\left|x_{2}\right|+\left|x_{3}\right|+\left|x_{4}\right|\right)$

The effect of the $x_{6}$ term is to generate a synchronizing term that serves to lock the phase between $x_{1}$ and $-x_{3}$. A typical transient response is shown in Fig. 4.

\section{COMPARISON WITH OPEN-LOOP APPROXIMATE INVERSES}

The reachability results of [7] show that by one measure, sinusoids are the most efficient waveforms for altering the $X$-like terms in our basic system. Their use efficiently generates the necessary area terms. The work of Liu and Sussmann [15] establishes a very general result on tracking with nonholonomic system. One might say they show how the generation of area by sinusoids can be separated from the generation of position. It was suggested in [11] that the idea of an approximate inverse system could be useful in studying stabilization, approximate 


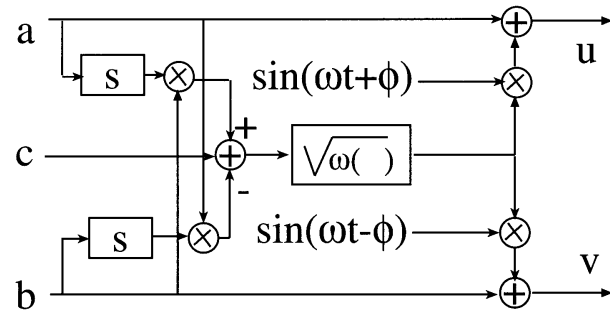

Fig. 5. Block diagram of an approximate inverse for the basic system.

linearization, etc. Krishnaprasad et al. [16] and Morgansen in [17] have subsequently extended the inverse system idea in a number of directions.

The idea behind the approximate inverse is to provide an adjustable level of modulation on the input signals so as to steer the state variables of the system

$$
\begin{aligned}
& \dot{x}_{1}(t)=-x_{1}(t)+u_{1}(t) \\
& \dot{x}_{2}(t)=-x_{2}(t)+u_{2}(t) \\
& \dot{x}_{3}(t)=-x_{3}(t)+x_{1}(t) u_{2}(t)-x_{2}(t) u_{1}(t)
\end{aligned}
$$

along a desired path. Suppose we have low frequency inputs $a, b, c$ and we want to find a mapping $\Phi:(a, b, c) \rightarrow(u, v)$ such that $(u, v)$ causes the system to track these functions in the sense that the vector $(x-a, y-b, z-c)$ is small. The plan for constructing the approximate inverse is to think of $u$ and $v$ as being the sum of two parts, a part that uses the lower frequency portion of the power spectrum and can be taken to be $u=\dot{a}, v=\dot{b}$ and a second part, occupying a frequency band well above that which is needed for $a$ and $b$ which will drive $x_{3}$. The latter will be shaped by a combination of amplitude and phase modulation in accordance with the function $c-(a \dot{b}-b \dot{a})$. We may think of this last expression as being " $(x, y)$-corrected" in that we have subtracted off the influence of the area defined by the desired path of $x$ and $y$.

Of course, we can add high frequency terms to $u$ and $v$ in different ways using different types of modulation. Because the dynamics associated with $x$ and $y$ will naturally suppress higher frequency effects more than lower frequency effects, it is useful to think in terms of the ratio of the modulation frequency and the reciprocal of the time constant associated with the $x-y$ dynamics. Modulation becomes much less effective if the frequency falls below $\omega_{0}=1 / T$. Thinking in terms of the types of applications mentioned in Section I, it is clear that one can achieve more movement in the $x_{3}$-direction if the frequency is increased along with the magnitude of the modulation term. (Faster walking is achieved by lengthening the stride and increasing the steps per unit time).

This reasoning, together with some natural choices of modulation, leads to open-loop approximate inverses of the general form shown in Fig. 5. This is discussed in much more detail in [10].

Of course, the purpose of the approximate inverse is to create a precompensator for the nonholonomic system that makes control or path planning easy. Thus the cascade of the inverse with the nonholonomic system is of central interest. The open-loop

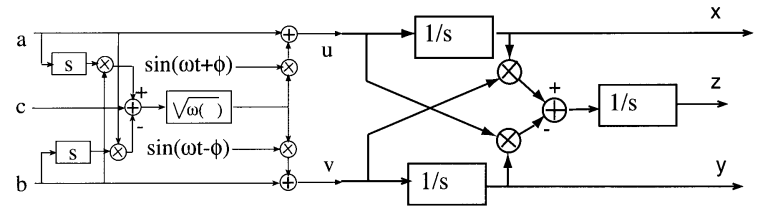

Fig. 6. Open-loop approximate inverse cascade as an alternative to the system of Fig. 2.

cascade, shown in Fig. 6, can be contrasted with the feedback form of inversion shown in Fig. 2. The important distinction here is the difference between situations in which the oscillations are generated internally verses those using the insertion of a periodic signal from some other source.

\section{CONCLUSION}

We have argued that the appearance of time-periodic phenomena in both man made and biological systems can often be traced to nonintegrable effects of the type that arises in nonlinear controllability. This point of view unifies many diverse phenomena found in engineering and nature. We investigate here the analog of the regulator problem in this context, not from the point of view of optimal control, but rather from a more classical point of view. We define stabilizing control laws for large classes of systems, drawing a distinction between the cases where regulation involves both frequency and amplitude adjustment and the case where amplitude adjustment is used alone. The treatment of the higher dimensional cases involves analysis on the space of skew-symmetric matrices. In particular, Theorem 3, which addresses a rather general class of problems, makes use of the idea of a maximal commuting subalgebra and succeeds in establishing a result on controlling the phases between synchronous modes of oscillation. We also discuss nonsinusoidal oscillations and make use of them in a model of biped locomotion.

\section{ACKNOWLEDGMENT}

The author would like to thank K. Morgansen for help in exploring the literature on locomotion patterns. He would also like to thank the anonymous referees for their helpful remarks.

\section{REFERENCES}

[1] A. Shapere and F. Wilczek, Geometric Phases in Physics. Singapore: World Scientific, 1989

[2] R. W. Brockett, "On the rectification of vibratory motion," Sensors Actuators, vol. 20, pp. 91-96, 1989.

[3] G. Kron, "Non-Riemannian dynamics of rotating electrical machinery," J. Math. Phys., vol. 13, pp. 103-194, 1934.

[4] R. Severns and E. Bloom, DC to DC Converters. New York: Van Nostrand, 1987.

[5] I. M. Gelfand et al., Models of the Structural-Functional Organization of Certain Biological Systems. Cambridge, MA: MIT Press, 1971.

[6] B. Gaveau, "Principe de Moindre Action, Propagationde la Chaleur et Estemées Sous Elliptiques sur certains Groupes Nilpotents," Acta Math., vol. 139, pp. 94-153, 1977.

[7] R. W. Brockett, "Control theory and singular Riemannian geometry," in New Directions in Applied Mathematics, P. Hilton and G. Young, Eds. New York: Springer-Verlag, 1981, pp. 11-27.

[8] G. Brown, "Intrinsic factors in the act of progression of the mammal," in Proc. Royal Soc. London, vol. 84, 1911.

[9] T. A. McMahon, "Using body size to understand the structural design of animals: quardupedal locomotion," J. Appl. Physiol., vol. 39, pp. 619-627, 1975. 
[10] K.Kurt von Euler, "Pattern generation during breathing," in The Motor System in Neurobiology, E. Evarts, S. Wise, and D. Bousfield, Eds. Amsterdam, The Netherland: Elsevier, 1985

[11] R. W. Brockett, "Characteristic phenomena and model problems in nonlinear control," in Proc. 1996 IFAC Congr., vol. G, 1996, pp. 135-140.

[12] H.Hans Samelson, Notes on Lie Algebras. New York: Springer-Verlag, 1990.

[13] R. W. Brockett, "Lie algebras and Lie groups in control theory," in Geometric Methods in System Theory, D. Q. Mayne and R. W. Brockett, Eds. Dordrecht, The Netherlands: Reidel, 1973, pp. 43-82.

[14] M. J. Coleman and P. Holmes, "Motions and stability of a piecewise holonomic system: The discrete Chaplygin Sleigh," Regular Chaotic Dyna., vol. 4, no. 2, pp. 1-23, 1999.

[15] W.Wenshang Liu and H. J. Sussmann, "Limits of highly oscillatory controls and the approximation of general paths by admissible trajectories," in Proc. 30th IEEE Conf. Decision Control, 1991, pp. 437-442.

[16] H. K. Struemper and P. S. Krishnaprasad, "On approximate inversion and feedback stabilization for systems on matrix Lie groups," in Proc. Amer. Control Conf., Philadelphia, PA, 1997, pp. 2576-2580.

[17] K. Morgansen, "Temporal patterns in learning and control," Ph.D. dissertation, Harvard Univ., Cambridge, MA, 1999.
Roger W. Brockett (S'62-M'63-SM'73-F'74) received the B.S., M.S., and Ph.D. degrees from Case Western Reserve University, Cleveland, OH, in 1960 , 1962, and 1964, respectively.

$\mathrm{He}$ is the An Wang Professor of Electrical Engineering and Computer Science in the Division of Applied Science at Harvard University, Cambridge, MA He taught for six years in the Electrical Engineering Department at the Massachusetts Institute of Technology, Cambridge, before joining the Harvard Faculty in 1969. He has contributed extensively to the theory of automatic contro with work on stability, nonlinear control, feedback linearization, system identification, nonlinear estimation, pole placement, hybrid systems, and robotics More recently, his work has involved problems arising in the study of intelligent machines. Areas of particular interest include the problem of motion control, and the investigation of new paradigms involving the concept of attention in high-data rate, sensory-rich environments.

Dr. Brockett has been recognized by the American Automatic Control Council and the IEEE through their Richard Bellman Award and the Control System Science and Engineering Award. He has received the Reid Prize from the Society of Industrial and Applied Mathematics. He has served on a variety of National Research Council Panels and is a Member of the National Academy of Engineering. 